\title{
ORIGINAL ARTICLE 50 years follow-up on plasma creatinine levels after spinal cord injury
}

\author{
M Elmelund ${ }^{1}$, PS Oturai ${ }^{1}$ and F Biering-Sørensen ${ }^{2,3}$
}

\begin{abstract}
Study design: Retrospective chart review.
Objectives: To investigate the role of plasma creatinine ( $p$-creatinine) in monitoring renal deterioration in patients up to 50 years after spinal cord injury (SCI).

Setting: The Clinic for Spinal Cord Injuries, Rigshospitalet, Denmark.

Methods: A total of 119 patients with a traumatic SCl during the years 1944-1975 were included in the study. P-creatinine measurements, results from renography and glomerular filtration rate (GFR) measured with ${ }^{51} \mathrm{Cr}$-EDTA clearance were obtained from medical records and analyzed using a linear mixed model and linear regression analyses.

Results: When compared with median p-creatinine level in the first 5-year period after injury, the level of p-creatinine was stable throughout the first 30 years and decreased significantly after the 30th until 45th year post injury. Only patients with a functional distribution outside the $30-70 \%$ limits on renography or a relative GFR $\leqslant 51 \%$ of that expected had a significantly elevated level of p-creatinine. Significance was not found for patients with a distribution outside the 40-60\% limits on renography or relative GFR $\leqslant 75 \%$. By comparing Cr-EDTA clearance and p-creatinine in terms of exceeding the upper reference level, p-creatinine revealed $17 \%$ sensitivity, $100 \%$ specificity, $100 \%$ positive predictive value and $73 \%$ negative predictive value as a diagnostic test for renal deterioration defined as GFR $\leqslant 75 \%$.
\end{abstract}

Conclusion: P-creatinine decreases over time in patients with $\mathrm{SCl}$ with a level below the upper reference limit and is a poor detector of early renal deterioration in patients with $\mathrm{SCl}$.

Spinal Cord (2014) 52, 368-372; doi:10.1038/sc.2014.24; published online 11 March 2014

Keywords: creatinine; glomerular filtration rate; kidney function; renography; spinal cord injury

\section{INTRODUCTION}

Individuals who have sustained a spinal cord injury (SCI) are at increased risk of developing renal insufficiency, because of neurogenic bladder dysfunction. For this reason, SCI patients are recommended to participate in a lifelong follow-up program of the renal function. Despite numerous attempts to evaluate the follow-up program, the best method to monitor the renal function in this patient group has not yet been established. ${ }^{1}$ A widely used method is the repeated measurement of plasma creatinine ( $\mathrm{p}$-creatinine), ${ }^{2}$ and equations like the Cockcroft and Gault or MDRD (modification of diet in renal disease) equations have been derived to estimate glomerular filtration rate (GFR) from p-creatinine, age, weight and gender. ${ }^{3,4} \mathrm{P}$-creatinine is primarily derived from the metabolism of creatine and phosphocreatine in muscles. ${ }^{5}$ Patients with SCI often have a reduced muscle mass because of diffuse muscle atrophy and for this reason the level of p-creatinine is often decreased in such patients. ${ }^{2}$ The hypothesis that $\mathrm{p}$-creatinine overestimates the renal function in this patient group has been demonstrated in several studies since the 1980 s, ${ }^{6-9}$ but despite this p-creatinine is still a commonly accepted method to monitor renal function in SCI patients. Recognizing this, we wanted to investigate if there was a trend in the level of p-creatinine over a 50-year follow-up period and how well p-creatinine correlated with renal deterioration. To our knowledge, only three studies have investigated the relationship between $\mathrm{p}$-creatinine and renal function monitored by ${ }^{51} \mathrm{Cr}$-EDTA or ${ }^{99 \mathrm{~m}} \mathrm{Tc}$ DTPA clearance, which is considered the gold standard. ${ }^{2,10,11}$ Of these three studies, none of them investigated $\mathrm{p}$-creatinine over time and two of them only comprised 31 and 36 patients, respectively.

\section{MATERIALS AND METHODS}

\section{Study population}

This retrospective study originally included 154 patients who were registered with a traumatic SCI between the years 1944 and 1975 and who initially were admitted to the Clinic of Spinal Cord Injuries in Hornbæk, Denmark.

Data regarding patient demographic, neurological level and completeness of injury according to Frankel et al. ${ }^{12}$ and the primary bladder emptying method $^{13}$ were obtained from medical records, as well as all p-creatinine, renography and GFR measurements by using ${ }^{51} \mathrm{Cr}$-EDTA clearances. Of the 154 patients, there were 13 patients with missing medical records and 7 patients who had not sustained an actual traumatic SCI. Patients who were not followed up with minimum one renography or GFR (15 patients) were excluded from the study. Thus, 119 patients were included in the final analyses as shown in Figure 1.

None of the patients had diabetes at the time of SCI. There were six individuals with only one functional kidney: two of them because of a traumatic kidney damage in relation to the SCI and four because of a complete nephrectomy post injury.

${ }^{1}$ Department of Clinical Physiology, Nuclear Medicine and PET, Rigshospitalet, Copenhagen, Denmark; ${ }^{2}$ Centre for Spinal Cord Injured, Rigshospitalet and Glostrup Hospital, Copenhagen, Denmark and ${ }^{3}$ Faculty of Health and Medical Sciences, University of Copenhagen, Copenhagen, Denmark

Correspondence: Dr M Elmelund, Department of Clinical Physiology, Nuclear Medicine and PET, Rigshospitalet, Blegdamsvej 9, Copenhagen 2100, Denmark.

E-mail: marleneelmelund@hotmail.com

Received 6 October 2013; revised 24 January 2014; accepted 7 February 2014; published online 11 March 2014 


\section{Procedures}

After the SCI, patients who accepted the follow-up program were offered a routine check about every second year lifelong at the Clinic for Spinal Cord Injuries. In the early years, follow-up consisted of p-creatinine measurement and X-ray of the abdomen or intravenous pyelography as a minimum, but since 1980 renography was performed on a regular basis and, when considered necessary, GFR was measured using plasma clearance determination of a radioactive tracer.

The majority of renographies were performed with ${ }^{99 \mathrm{~m}}$-Tc-labelled mercaptoacetyltriglycine three, but renography with dimercaptosuccinic acid, diethylentriamine pentaacetic acid (DTPA) and ${ }^{131}$ I-hippuran was also accepted in this study. In consensus with previous studies, ${ }^{14,15}$ renography was considered moderately abnormal when there was a split renal function $\leqslant 40 \%$ in one kidney and considered more severely abnormal when there was a split renal function $\leqslant 30 \%$ in one kidney.

GFR was measured using a single injection technique with a radioactive isotope-marked tracer, in most cases with ${ }^{51} \mathrm{Cr}$-EDTA, and converted to an age and gender-adjusted relative GFR. ${ }^{16}$ Relative GFR $\leqslant 75 \%$ was considered moderately abnormal and $\leqslant 51 \%$ more severely abnormal. In this study, patients with at least one abnormal renography and/or GFR were categorized with moderate or more severe renal affection. Individuals with only one functional kidney since the time of injury and with no abnormal GFR measurements were classified as having normal renal function.

P-creatinine values were registered and if necessary converted from the unit $\mathrm{mg} \mathrm{dl}{ }^{-1}$ to $\mu \mathrm{moll}^{-1}$ by dividing by 0.0113 . When there was more than one p-creatinine measurement from the same year post injury, the highest value was registered. P-creatinine measurements (with a maximum of one value per year) were further assembled in the form of individual median p-creatinine values for the periods $0-5$ years post injury (YPI), 6-10 YPI, and so on, and statistical analyses were performed on the logarithmical values of median 5 -year p-creatinine levels. The upper reference level for a normal p-creatinine value was $105 \mu \mathrm{moll}^{-1}$ for males and $90 \mu \mathrm{moll}^{-1}$ for females, which is the official upper reference level since 2003 in our hospital and is the lowest upper reference level used in the follow-up period.

We also wanted to investigate how well the level of p-creatinine correlated with the renal function; thus, a linear regression analysis on relative GFR and p-creatinine measurements from the same year post injury was done. When a patient had more than one GFR and p-creatinine measurement from the same year, the lowest GFR and its corresponding p-creatinine level were selected. In order to get the best linear fit and to make the results comparable to previous studies, the analysis was performed on the reciprocal value, $1 / \mathrm{p}$-creatinine. In addition, p-creatinine as a diagnostic test was evaluated by comparing p-creatinine measurements with GFR measurements.

All statistical analyses were performed using SPSS and Microsoft Excel 2011 software packages. $P$-values less than 0.05 were considered significant and $P$-values were calculated with Fisher's exact test. To characterize the trend in

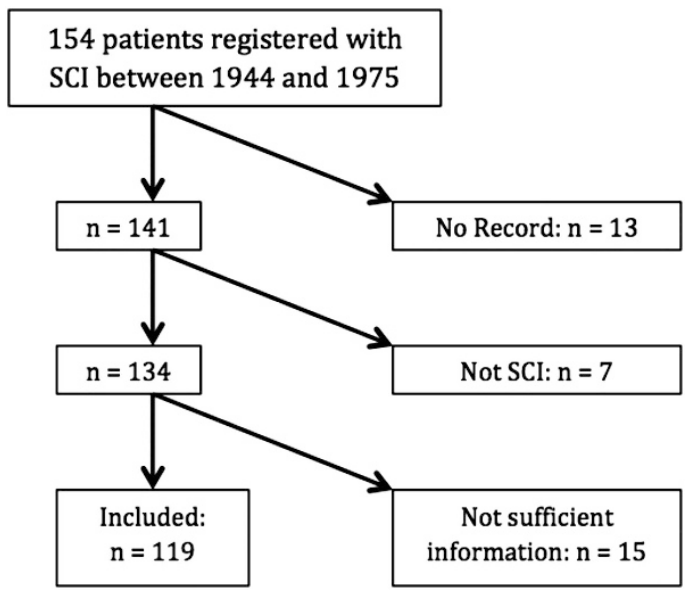

Figure 1 Flow diagram for patients in the study. SCl: Spinal cord injury. p-creatinine over time, a linear mixed module with individual median 5-year p-creatinine values was used and illustrated as mean \pm normal range, which is a $95 \%$ prediction interval of the population. Correlation analysis was performed and Pearson's correlation coefficient, $r$, and determination coefficient, $r^{2}$, were calculated.

\section{RESULTS}

Study population

Patient characteristics and primary bladder emptying methods are shown in Tables 1 and 2.

Table 1 Characteristics of patients with spinal cord injuries, median (inter quartile range), total numbers (\%)

$\begin{array}{lc}\text { No. of patients } & 119 \\ \text { Gender } & 16(13 \%) \\ \quad \text { Female } & 103(87 \%) \\ \quad \text { Male } & 21(18-25) \\ \text { Age of injury (years) } & 40(36-44) \\ \text { Follow-up (years post injury) } & \end{array}$

$\begin{array}{lc}\text { Level of neurological injury } & \\ \text { Cervical } & 56(47 \%) \\ \text { Thoracic } & 48(40 \%) \\ \text { Lumbal } & 15(13 \%) \\ \text { Sacral } & 0\end{array}$

Completeness (Frankel grade)

$49(41 \%)$

$28(24 \%)$

$14(12 \%)$

C

$25(21 \%)$

$3(2 \%)$

$43(36 \%)$

\section{Cause of death ${ }^{\mathrm{a}}$}

Pneumonia/other respiratory causes $11(26 \%)$

Cancer $6(14 \%)$

Septicemia $\quad 4(9 \%)$

Heart disease $3(7 \%)$

Injuries $2(5 \%)$

Urinary system diseases $\quad 1(2 \%)$

Digestive system diseases $\quad 1(2 \%)$

Arteries/pulmonary circulation diseases $\quad 1(2 \%)$

Unknown/ill-defined causes

$14(33 \%)$

aCause of death according to death certificate or medical records.

Table 2 Primary bladder emptying method at time of discharge from initial admission at the Centre for Spinal Cord Injuries and at the last follow-up

\begin{tabular}{lcc}
\hline & Discharge & Last follow-up \\
\hline Bladder reflex triggering & $76(64 \%)$ & $26(22 \%)$ \\
Bladder expression & $26(22 \%)$ & $4(3 \%)$ \\
Normal voiding & $7(6 \%)$ & $5(4 \%)$ \\
Indwelling catheter & $4(3 \%)$ & $39(33 \%)$ \\
Clean intermittent catheterization & 0 & $40(34 \%)$ \\
lleal Conduit a.m. Bricker & 0 & $5(4 \%)$ \\
Unknown & $6(5 \%)$ & 0
\end{tabular}

Each patient may use more than one method. Bladder expression: abdominal pressure and Credé manoeuvre. 
The primary bladder emptying methods have changed over time. Reflex triggering and bladder expression were the most frequently applied methods at the time of discharge after the initial post-injury rehabilitation, whereas clean intermittent catheterization and indwelling catheter (urethral or suprapubic) were the most frequently applied methods at the last follow-up.

\section{P-creatinine over time}

The average number of years with a p-creatinine measurement per patient was 17 years $( \pm 7.4)$, mean ( \pm s.d.). Figure 2 shows no increase in the mean of 5 -year median p-creatinine values over time for both males and females, and Table 3 confirms that the means of 5-year median p-creatinine values are stable in the first 30 years after SCI and show a significant decrease when comparing 0-5 YPI with 31-45 YPI. The last period of follow-up was 46-50 YPI, after which the means became unreliable because of small sample sizes. The means of p-creatinine never exceeded the upper reference level of p-creatinine (male $105 \mu \mathrm{moll}^{-1}$ and female $90 \mu \mathrm{moll} \mathrm{l}^{-1}$ ), and when looking at all the p-creatinine samples 73 patients (61\%) never exceeded the upper reference limit, 24 patients $(20 \%)$ exceeded the limit one time and 22 patients (19\%) exceeded the limit more than one time. Six male patients experienced a p-creatinine level of $200 \mu \mathrm{moll}^{-1}$ or more: five of them with concomitant severe renal affection with functional distribution outside $30-70 \%$ on renography (four patients), r-GFR51 (one patient) and/or in dialysis treatment (two patients). The last patient did not have renal deterioration according to renography or GFR measurements and was hospitalized with encephalitis simultaneous with a p-creatinine level of $224 \mu \mathrm{moll}^{-1}$.
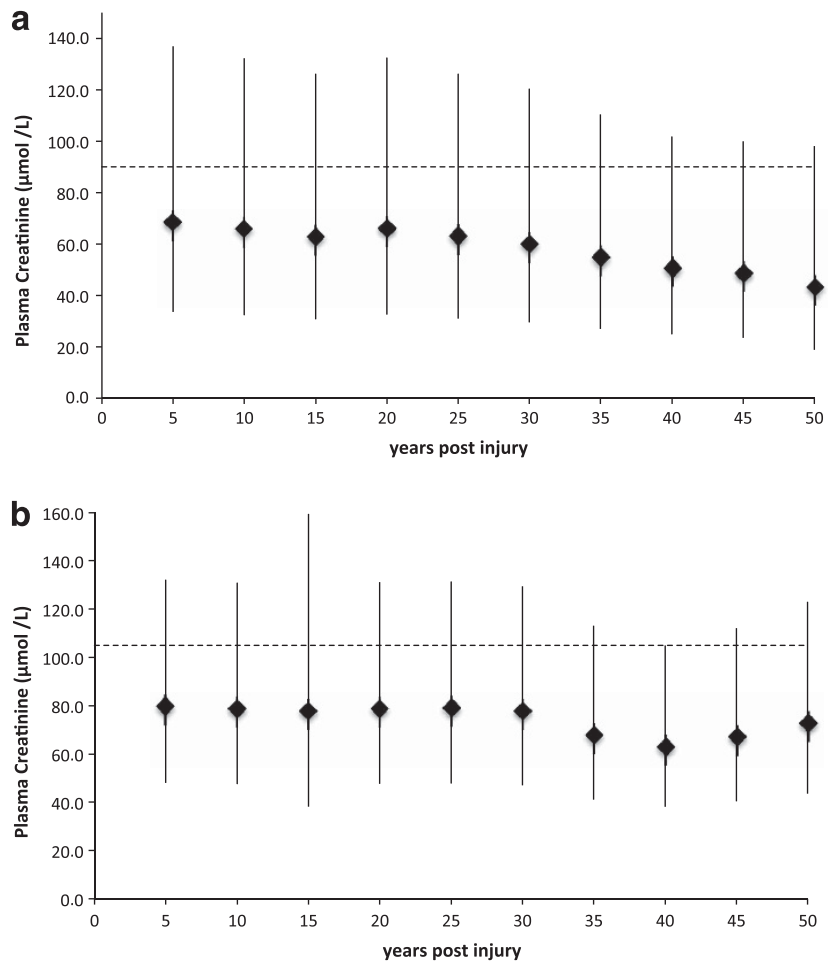

Figure 2 Mean ( $\pm 95 \%$ normal ange) $p$-creatinine of individual median p-creatinine values in 5 -year periods for (a) males $(n=103)$ and (b) females $(n=16)$, with the upper limit of reference interval for $p$-creatinine (males $105 \mu \mathrm{moll}^{-1}$ and females $90 \mu \mathrm{mol} \mathrm{I}^{-1}$ ).

\section{P-creatinine and renal function}

In the linear mixed module analysis, we found no significant difference in the level of individual median $\mathrm{p}$-creatinine in 5-year periods between patients with moderate renal affection and patients with normal renal function. Patients with more severe renal affection had a significantly higher level of individual 5-year median p-creatinine compared with patients with normal or moderate renal affection, with a difference of $12 \%$ (95\% CI: 7-19\%, $P<0.0001)$. When comparing patients with all p-creatinine values within the normal reference interval with patients with one or more p-creatinine values exceeding the reference level, there was a significant difference with regard to severe renal affection $(P 0.04)$ but not with regard to moderate renal affection $(P=0.13)$.

Figure 3 shows the relationship between relative GFR and p-creatinine, based on the results from 79 patients with a GFR and a p-creatinine measurement from the same YPI ( $38 \pm 8$, means \pm s.d.). With renal deterioration defined by the gold standard Cr-EDTA clearance as $\mathrm{r}$-GFR $\leqslant 75 \%$, the use of $\mathrm{p}$-creatinine as a diagnostic test can be evaluated. We considered p-creatinine as a positive test for renal deterioration when exceeding the upper reference level. Of the 79 patients, there were 4 true positive, 20 false negative, 0 false positive and 55 true negative tests, revealing $17 \%$ sensitivity, $100 \%$ specificity, $100 \%$ positive predictive value and $73 \%$ negative predictive value of $\mathrm{p}$-creatinine as a diagnostic test for renal deterioration.

Figure 4 shows the relationship between relative GFR and $1 / \mathrm{p}$-creatinine, and a significant linear relationship between GFR and $1 / \mathrm{p}$-creatinine for the 69 male patients was found with Pearson's coefficient $r=0.448$ and a determination coefficient $r^{2}=0.201$ ( $P$-value $<0.0001)$. There was a non-significant relationship for the 10 female patients with $r=0.275$ and $r^{2}=0.075(P$-value $=0.443)$.

\section{DISCUSSION}

The aim of the present study was to investigate the trend of p-creatinine over time in patients with SCI and to examine if p-creatinine is useful in predicting renal deterioration. This study indicates that SCI patients have a steady level of p-creatinine during the first 30 years and a significant decrease after the 30th YPI. This finding is consistent with previous studies showing no increase in p-creatinine over time. ${ }^{7,9,14,15,17}$ The longest follow-up in previous

Table 3 Change in means of 5-year median Plasma creatinine over time after the spinal cord injury

\begin{tabular}{|c|c|c|c|c|c|}
\hline \multirow{2}{*}{$\begin{array}{l}\text { Time interval } \\
(Y P I)\end{array}$} & \multirow{2}{*}{$\begin{array}{l}\text { No. of } \\
\text { patients }\end{array}$} & \multirow{2}{*}{$\begin{array}{l}\text { Change from } \\
\text { period } 0-5 \\
\text { YPI (\%) }\end{array}$} & \multicolumn{2}{|c|}{$\begin{array}{c}\text { Change from period 0-5 } \mathrm{YPI} \text {, } \\
95 \% \text { confidence interval }\end{array}$} & \multirow{2}{*}{ P-value } \\
\hline & & & $\begin{array}{c}\text { Lower } \\
\text { bound (\%) }\end{array}$ & $\begin{array}{c}\text { Upper } \\
\text { bound (\%) }\end{array}$ & \\
\hline $0-5$ & 98 & 0 & & & \\
\hline $6-10$ & 91 & -0.7 & -7.7 & 6.9 & 0.852 \\
\hline $11-15$ & 79 & -2.9 & -10.1 & 5 & 0.455 \\
\hline $16-20$ & 86 & -1.1 & -8.4 & 6.4 & 0.754 \\
\hline $21-25$ & 90 & -1.4 & -8.4 & 6.1 & 0.703 \\
\hline $26-30$ & 96 & -3.4 & -10.3 & 3.8 & 0.339 \\
\hline $31-35$ & 85 & -14.9 & -21.2 & -8.4 & $<0.001$ \\
\hline $36-40$ & 78 & -21.1 & -27.1 & -14.9 & $<0.001$ \\
\hline $41-45$ & 39 & -16.4 & -24.1 & -8.2 & $<0.001$ \\
\hline $46-50$ & 16 & -9.2 & -20.9 & 4 & 0.162 \\
\hline
\end{tabular}

Abbreviation: YPI, years post injury. 
studies was 18.7 YPI, ${ }^{14}$ and only one study with maximum 16 years follow-up found a significant decrease in p-creatinine over time. ${ }^{7}$ To our knowledge, our study has the longest follow-up period of 50 YPI.

In normal individuals, GFR declines with aging, with an average decrease of $10 \mathrm{ml} \mathrm{min}^{-1}$ per $1.73 \mathrm{~m}^{2}$ per decade after 30 years of age. Meanwhile, the total muscle mass declines with increasing age, and together these two changes result in a normal to slightly decreased p-creatinine concentration in older individuals. ${ }^{3}$ The fact that this study shows a steady level and later a significant decrease in p-creatinine over time could be a reflection of an increasing age in the patient population, perhaps accompanied by a more extensive loss of muscle mass over time in patients with a SCI compared with normal individuals. But, most importantly, no general increase in p-creatinine over time was found, despite the fact that SCI patients are at increased risk of developing renal insufficiency. ${ }^{2}$

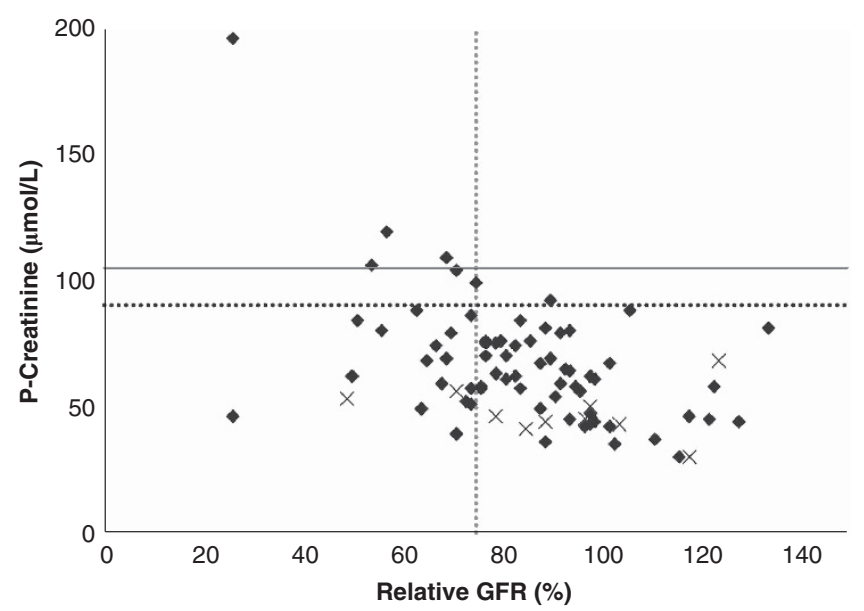

Figure 3 Plots of $r$-GFR (\% of normal reference value) versus $p$-creatinine for 79 spinal cord-injured patients: 69 male patients $(\downarrow)$ and 10 female patients $(x)$ from the same year post injury: $38( \pm 8)$ mean $( \pm$ s.d. $)$. The upper reference level for P-creatinine is $105 \mu \mathrm{moll}^{-1}$ for males (horizontal solid line) and $90 \mathrm{umoll}^{-1}$ for females (horizontal dotted line). The lower reference level for relative GFR is $75 \%$ (vertical dotted line).
The present study found that only patients with severe renal affection on renography $(30-70 \%$ or more abnormal) or GFR measurement (relative GFR $\leqslant 51 \%$ ) would have a higher level of $\mathrm{p}$-creatinine and $\mathrm{p}$-creatinine values exceeding the upper reference limit, whereas this could not be detected in patients with less severe deterioration of renal function.

Other studies have investigated the correlation between $1 / \mathrm{p}$-creatinine and GFR expressed with Pearson's correlation coefficient $(r)$. Thomassen et al. ${ }^{10}$ found a non-significant correlation between GFR measured with ${ }^{51} \mathrm{Cr}$-EDTA clearance and 1/s-creatinine in 31 SCI patients, with Pearson's correlation coefficient $r=0.26$, and Jenkins et al. ${ }^{18}$ found a non-significant correlation between GFR measured with creatinine clearance and $1 / \mathrm{s}$-creatinine for 20 male SCI patients, with $r=0.29$. The fact that our study included more SCI male patients $(n=69)$ than the two mentioned above may explain the significant correlation found in our study for the male patients. In comparison we found a stronger correlation ( $r=0.448)$, but the correlation is not strong, as $r$ is not close to 1 , and only $20 \%$ of the variation in $1 / \mathrm{p}$-creatinine can be explained by a change in GFR $\left(r^{2}=0.20\right)$, indicating that the results of p-creatinine measurements correspond poorly to the results of GFR measurements. A $17 \%$ sensitivity of p-creatinine as a diagnostic test in terms of p-creatinine values exceeding the upper reference level underlines the poor use of p-creatinine in diagnosing renal deterioration.

A few studies have described p-creatinine compared with renal function within the last 15 years, ${ }^{2,10,11,17-20}$ most of them using creatinine clearance as a measure of the renal function. Only three of the papers used the gold standards ${ }^{51} \mathrm{Cr}$-EDTA clearance ${ }^{10,11}$ or ${ }^{99 \mathrm{~m}}$ Tc-DTPA clearance. ${ }^{2}$ They all concluded that s-creatinine underestimated the renal deterioration, except for one study, Sepahpanah et al., ${ }^{17}$ which concluded that s-creatinine was better in monitoring renal function than creatinine clearance.

A newer marker, cystatin $\mathrm{C}$, has shown great potential in detecting renal deterioration in patients with SCI ${ }^{10,11,18}$ It is produced by most nucleated cells at a stable rate and is, most importantly, not affected by the level of muscle mass, gender or age, ${ }^{10}$ which makes it a promising test in this patient population and hence it should probably substitute $\mathrm{p}$-creatinine in the future.

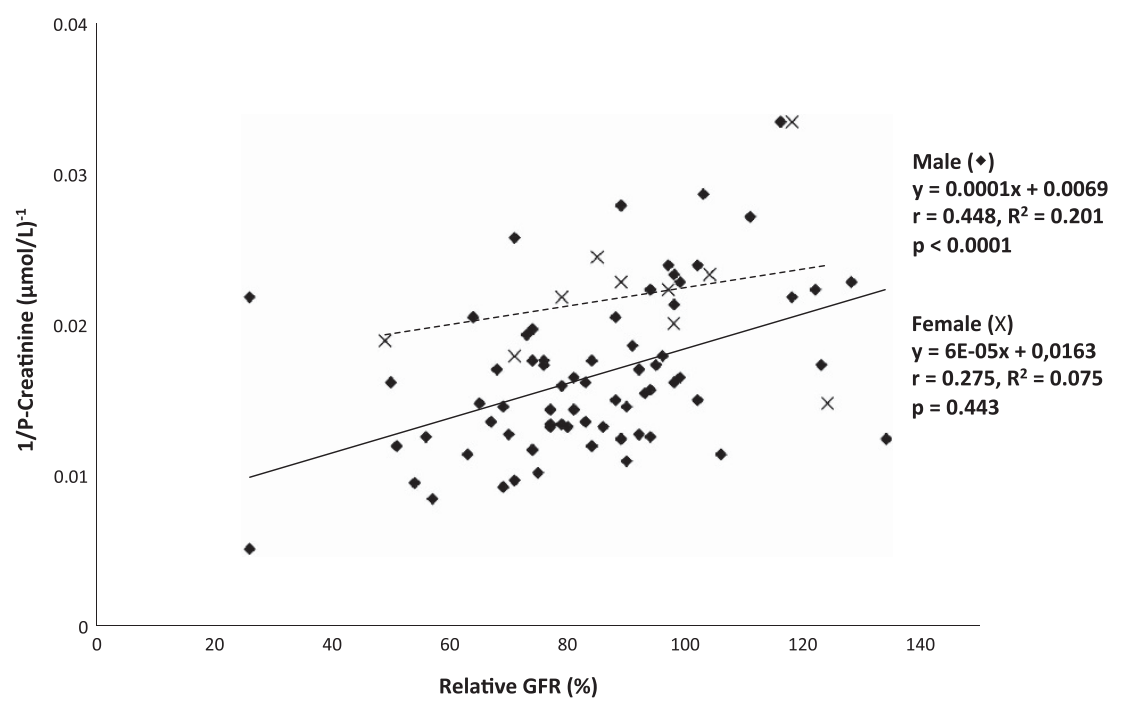

Figure 4 Linear regression plots of r-GFR (\% of normal reference value) versus 1/p-creatinine for 79 spinal cord-injured patients: 69 male patients ( $\$$ ) with solid line and 10 female patients $(x)$ with striped line, from the same year post injury: $38( \pm 8)$, mean ( \pm s.d.). 


\section{Limitations}

An important limitation to our retrospective study is the risk of missing data. Some patients did not attend the annual evaluations in many years after the injury and were then found again as a result of the additional follow-up effort. In this study 12 patients (10\%) were not followed with p-creatinine in the first 20 YPI.

A retrospective study like this one with the earliest SCI in 1944 and with investigations carried out in different hospitals may also have some limitations in terms of comparing the investigation results. The method of measuring p-creatinine has for instance changed throughout the years in Rigshospitalet, where the majority of the p-creatinine measurements have been performed. For most of the measurements the Jaffe method has been used, but after 2003 an enzymatic IDMS calibrated method, CREA plus Roche Modular Analytics with a lower reference level, was introduced. We have considered if this could result in an artificial decrease in the level of p-creatinine over time, but as the patient population had sustained the SCI over a 31-year period, the date of decreasing $\mathrm{p}$-creatinine values was spread out over the interval 28-59 YPI, and therefore this should not particularly affect our conclusion. Additionally, we only used the lowest and most conservative upper reference limit introduced in 2003 in our analyses to make sure we did not overlook any increase in the p-creatinine values.

Estimated GFR values can be calculated from p-creatinine measurements from the patient's weight, age and gender, using, for example, the MDRD equation, and the estimated GFR and measured GFR can be compared accurately, but there were no repeated measures of weight available in this study, and therefore we were unable to make GFR estimations.

In this study, the renal function was determined from the results of renography with regard to functional renal distribution and GFR measured with a radioactive marked tracer. The presence of one abnormal investigation categorized the patients in either moderate or more severe renal affection. This definition of renal deterioration has limitations though, as it does not include a potential improvement in later investigations.

\section{CONCLUSION}

The findings of this study support that p-creatinine is a poor detector of early renal deterioration, and until new tests like Cystatin $\mathrm{C}$ are further evaluated we recommend Cr-EDTA clearance as the gold standard in monitoring renal function in patients with SCI.

\section{DATA ARCHIVING}

There were no data to deposit.

\section{CONFLICT OF INTEREST}

The authors declare no conflict of interest.

\section{ACKNOWLEDGEMENTS}

The study has been supported by grants from the Lundbeck foundation. We thank Julie Forman, Associate Professor from the Department of Biostatistics, University of Copenhagen, for statistical assistance.

1 Cameron AP, Rodriguez GM, Schomer KG. Systematic review of urological followup after spinal cord injury. J Urol 2012; 187: 391-397.

2 MacDiarmid SA, McIntyre WJ, Anthony A, Bailey RR, Turner JG, Arnold EP. Monitoring of renal function in patients with spinal cord injury. BJU Int 2000; 85 : 1014-1018.

3 Perrone RD, Madias NE, Levey AS. Serum creatinine as an index of renal function: new insights into old concepts. Clin Chem 1992; 38: 1933-1953.

4 Levey AS, Coresh J, Greene T, Stevens LA, Zhang YL, Hendriksen S et al. Using standardized serum creatinine values in the modification of diet in renal disease study equation for estimating glomerular filtration rate. Ann Intern Med 2006; 145: 247-254.

5 Thomas L, Huber AR. Renal function-estimation of glomerular filtration rate. Clin Chem Lab Med 2006; 44: 1295-1302.

6 Sawyer WT, Hutchins K. Assessment and predictability of renal function in spinal cord injury patients. Urology 1982; 19: 377-380.

7 Mohler JL, Barton SD, Blouin RA, Cowen DL, Flanigan RC. The evaluation of creatinine clearance in spinal cord injury patients. J Urol 1986; 136: 366-369.

8 Mirahmadi MK, Byrne C, Barton C, Penera N, Gordon S, Vaziri ND. Prediction of creatinine clearance from serum creatinine in spinal cord injury patients. Paraplegia 1983; 21: 23-29.

9 Kuhlemeier KV, McEachran AB, Lloyd LK, Stover SL, Fine PR. Serum creatinine as an indicator of renal function after spinal cord injury. Arch Phys Med Rehabil 1984; 65: 694-697.

10 Thomassen SA, Johannesen IL, Erlandsen EJ, Abrahamsen J, Randers E. Serum cystatin $\mathrm{C}$ as a marker of the renal function in patients with spinal cord injury. Spinal cord 2002; 40: 524-528.

11 Erlandsen EJ, Hansen RM, Randers E, Petersen LE, Abrahamsen J, Johannesen IL. Estimating the glomerular filtration rate using serum cystatin $\mathrm{C}$ levels in patients with spinal cord injuries. Spinal cord 2012; 50: 778-783.

12 Frankel HL, Hancock DO, Hyslop G, Melzak J, Michaelis LS, Ungar GH et al. The value of postural reduction in the initial management of closed injuries of the spine with paraplegia and tetraplegia. Paraplegia 1969; 7: 179-192.

13 Biering-Sorensen F, Craggs M, Kennelly M, Schick E, Wyndaele JJ. International lower urinary tract function basic spinal cord injury data set. Spinal cord 2008; 46: 325-330.

14 Weld KJ, Wall BM, Mangold TA, Steere EL, Dmochowski RR. Influences on renal function in chronic spinal cord injured patients. J Urol 2000; 164: 14901493.

15 Biering-Sorensen F, Nielans HM, Dorflinger T, Sorensen B. Urological situation five years after spinal cord injury. Scand J Urol Nephrol 1999. 33: 157-161.

16 Brochner-Mortensen J, Jensen S, Rodbro P. Delimitation of plasma creatinine concentration values for assessment of relative renal function in adult patients. Scand J Urol Nephrol 1977; 11: 257-262.

17 Sepahpanah F, Burns SP, McKnight B, Yang CC. Role of creatinine clearance as a screening test in persons with spinal cord injury. Arch Phys Med Rehabil 2006; 87 524-528.

18 Jenkins MA, Brown DJ, lerino FL, Ratnaike SI. Cystatin C for estimation of glomerular filtration rate in patients with spinal cord injury. Ann Clin Biochem 2003; 40: 364-368.

19 Lee JP, Dang AT. Evaluation of methods to estimate glomerular filtration rate versus actual drug clearance in patients with chronic spinal cord injury. Spinal Cord 2011; 49: 1158-1163.

20 Chikkalingaiah KB, Grant ND, Mangold TM, Cooke CR, Wall BM. Performance of simplified modification of diet in renal disease and Cockcroft-Gault equations in patients with chronic spinal cord injury and chronic kidney disease. Am J Med Sci 2010; 339: 108-116. 